\title{
A RESOLVED RING OF DEBRIS DUST AROUND THE SOLAR ANALOG HD 107146
}

\author{
Stuartt Corder ${ }^{1,2}$, John M. Carpenter ${ }^{1}$, Anneila I. Sargent ${ }^{1}$, B. Ashley Zauderer ${ }^{3}$, Melvyn C. H. Wright ${ }^{4}$, \\ Stephen M. White ${ }^{3}$, David P. Woody ${ }^{5}$, Peter Teuben ${ }^{3}$, Stephen L. Scott $^{5}$, Marc W. Pound $^{3}$, Richard L. Plambeck ${ }^{4}$, \\ James W. Lamb ${ }^{5}$, Jin Koda ${ }^{1}$, Mark Hodges ${ }^{5}$, David Hawkins ${ }^{5}$, And Douglas C.-J. Bock ${ }^{6}$ \\ ${ }^{1}$ Division of Physics, Mathematics and Astronomy, California Institute of Technology, MS 105-24 Caltech, Pasadena, CA 91125, USA \\ 2 Jansky Fellow, Current Address Av. Apoquindo 3650, Piso 18, Las Condes, Santiago, Chile \\ ${ }^{3}$ Department of Astronomy, University of Maryland, College Park, MD 20742, USA \\ ${ }^{4}$ Department of Astronomy and Radio Astronomy Laboratory, University of California, Berkeley, CA 94720, USA \\ ${ }^{5}$ Owens Valley Radio Observatory, California Institute of Technology, P.O. Box 968, Big Pine, CA 93513, USA \\ ${ }^{6}$ Combined Array for Research in Millimeter-wave Astronomy, P.O. Box 968, Big Pine, CA 93513, USA \\ Received 2008 July 31; accepted 2008 November 12; published 2008 December 8
}

\begin{abstract}
We present resolved images of the dust continuum emission from the debris disk around the young (80-200 Myr) solar-type star HD 107146 with CARMA at $\lambda=1.3 \mathrm{~mm}$ and the CSO at $\lambda=350 \mu \mathrm{m}$. Both images show that the dust emission extends over an approximately $10^{\prime \prime}$ diameter region. The high-resolution ( $3^{\prime \prime}$ ) CARMA image further reveals that the dust is distributed in a partial ring with significant decrease in a flux inward of 97 AU. Two prominent emission peaks appear within the ring separated by $\sim 140^{\circ}$ in the position angle. The morphology of the dust emission is suggestive of dust captured into a mean motion resonance, which would imply the presence of a planet at an orbital radius of $\sim 45-75 \mathrm{AU}$.
\end{abstract}

Key words: circumstellar matter - planetary systems - stars: individual (HD 107146)

\section{INTRODUCTION}

Photometric surveys with IRAS, ISO, and the Spitzer Space Telescope have identified hundreds of main-sequence stars where the observed flux densities at mid-infrared wavelengths are brighter than the stellar photosphere (for a review, see Meyer et al. 2007). This "excess" infrared emission is generally attributed to small dust grains that are produced when planetesimals collide and fragment into smaller debris.

The gravitational interaction of any planets with the debris dust produces gaps and peaks in the dust spatial distribution that provides clues to the underlying planetary system (Liou \& Zook 1999). However, the dust emission in most debris systems remains unresolved, and the dust spatial distribution is often inferred by modeling the composite spectral energy distribution. In practice, such analyses yield ambiguous results, since simplified disk geometries are assumed, and the inferred dust spatial distribution depends sensitively on the assumed grain composition, porosity, and size.

A few nearby debris disks have been resolved that provide direct information on the dust spatial distribution. Scattered light images at visible and near-infrared images often show smooth azimuthal distributions, frequently distributed in ringlike geometries (Kalas et al. 2004, 2005; Schneider et al. 2006). Resolved millimeter-wavelength images also show rings of material, but with more clumpy distributions of debris emission (Holland et al. 1998; Greaves et al. 1998, 2005; Koerner et al. 2001; Wilner et al. 2002; Maness et al. 2008). These clumps have been interpreted as signposts of planetary systems where the gravitational interactions trap the dust in mean motion resonances (Wilner et al. 2002; Wyatt 2003).

The debris disk around the G2V star HD 107146 (Jaschek 1978) has received special interest since it is a rare example of a resolved debris disk around a young (80-200 Myr; Williams et al. 2004) solar analog. At a distance of 28.5 pc (Perryman et al. 1997), the disk has been resolved in near-infrared scattered light (Ardila et al. 2004) and in the millimeter thermal continuum
(Williams et al. 2004; Carpenter et al. 2005). We present new (sub-)millimeter-wavelength images of the HD 107146 debris disk obtained with (CARMA) and the Caltech Submillimeter Observatory (CSO). The debris disk is clearly resolved in both data sets, with the CARMA image revealing a complex morphology not seen previously. We describe the observations in Section 2, present the resolved images in Section 3, and discuss the implications of these results in Section 4.

\section{OBSERVATIONS}

Continuum images of HD 107146 were obtained at wavelengths of $1.3 \mathrm{~mm}$ and $350 \mu \mathrm{m}$ using CARMA and the CSO, respectively. We adopted equatorial coordinates of $(\alpha, \delta)=$ $(12: 19: 06.50,+16: 32: 53.87)$ for equinox and epoch J2000, with a proper motion of $\left(\mu_{\alpha}, \mu_{\delta}\right)=(-175.65,-148.28)$ mas $\mathrm{yr}^{-1}$ (Perryman et al. 1997).

CSO observations were carried out between UT 2005 April 17 and 21, using the Submillimeter High Angular Resolution Camera II (SHARC II; Dowell et al. 2003) with the $350 \mu$ m filter. The median zenith opacity at $225 \mathrm{GHz}$ was 0.04 , which equates to an opacity of $\sim 1$ at $350 \mu \mathrm{m}$. Telescope pointing was checked on Callisto every $30 \mathrm{~min}$, which was $20^{\circ}$ away from HD 107146 at the time of the observations. Pointing was also checked on the fainter source $3 \mathrm{C} 273$, which is $15^{\circ}$ from HD 107146 , every hour. The pointing offsets as a function of time and elevation were interpolated in offline data processing and applied before co-adding the HD 107146 data. Absolute flux calibration was set from observations of Neptune assuming a total flux density of $92.6 \mathrm{Jy}$. We estimate a $1 \sigma$ calibration uncertainty of $15 \%$ from repeated observations of calibrators over the entire observing run. Images were produced using the Comprehensive Reduction Utility for SHARC II (CRUSH; Kovacs 2008). The "deep" data reduction mode in CRUSH was applied to both the calibrators and HD 107146.

CARMA observations were conducted on UT 2007 August 21, 2008 September 12, and 2008 September 14 in 


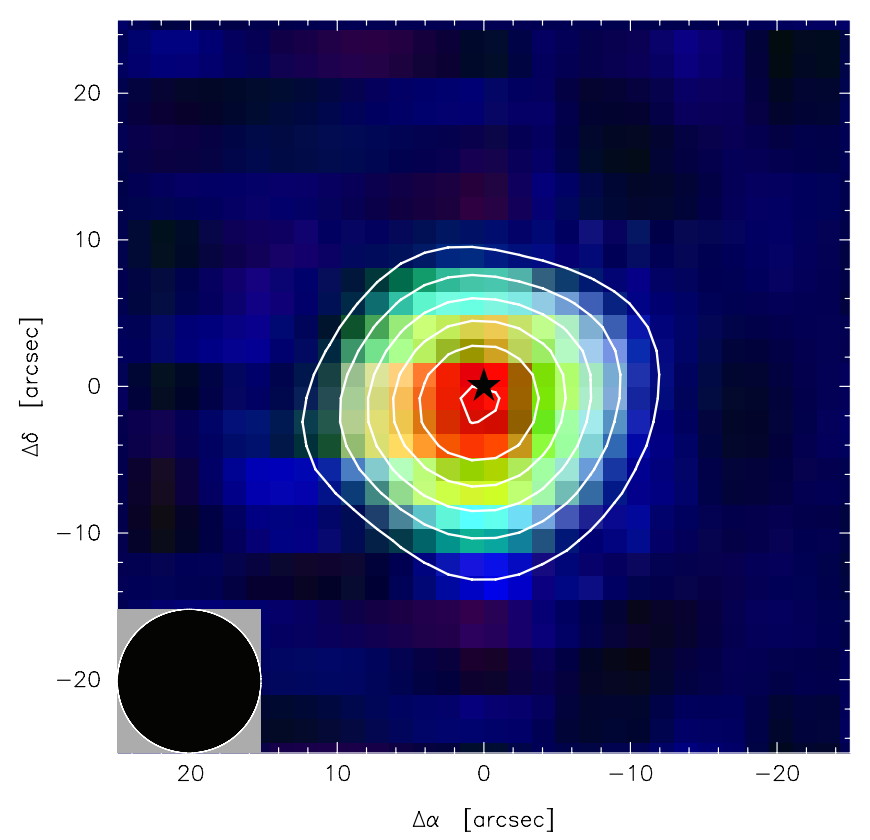

Figure 1. CSO $350 \mu \mathrm{m}$ continuum image of the HD 107146 debris disk Contours begin at $3 \sigma$ with increments of $5 \sigma$, where $\sigma=6 \mathrm{mJy}_{\text {beam }}{ }^{-1}$. The filled star at $(0,0)$ indicates the stellar position. The filled circle in the lower-left corner represents the 9!.8 resolution of the observations measured on the Callisto image.

the E-configuration, and on four days between UT 2008 March 3 and March 7 in the D-configuration. The E- and D-configurations span baselines of 6-66 $\mathrm{m}$ and 11-148 $\mathrm{m}$, respectively. Observations were conducted with the local oscillator (LO) set to a frequency of $227.25 \mathrm{GHz}$. Three correlator bands of $468 \mathrm{MHz}$ bandwidth each were placed at intermediate frequencies (IF) of $2.25,2.75$, and $3.25 \mathrm{GHz}$ to provide a total continuum bandwidth of $2.8 \mathrm{GHz}$ after combining the upper and lower sidebands. The quasar 3C273 was observed approximately every $20 \mathrm{~min}$ for amplitude, phase, and passband calibration. For the 2008 March and September observations, pointing was updated every $\sim 30 \mathrm{~min}$ using optical reference pointing (S. Corder et al. 2009, in preparation).

All reduction and imaging were performed using MIRIAD (Sault et al. 1995). Data were flux calibrated by observing Mars or MWC 349. For MWC 349, we assumed a flux density of $1.69 \mathrm{Jy}$ following the calibration adopted at the $\mathrm{PdBI}^{7}$ with an assumed uncertainty of $10 \%$ (Altenhoff et al. 1994). The nightto-night rms repeatability of the measured flux density of 3C273 is $2.4 \%$. We adopt a net calibration uncertainty of $15 \%$. The image was formed with natural weighting of the $u v$ visibilities and a Gaussian taper of FWHM = 1".6, and then deconvolved with the point spread function (i.e., the "dirty" beam) using a hybrid Högbom/Clark/Steer algorithm (Högbom 1974; Clark 1980; Steer et al. 1984). Since the emission is contained within a radius of $<5^{\prime \prime}$ from the phase center, no primary beam corrections were applied. The final image has a resolution of $3^{\prime \prime} .2 \times 2^{\prime \prime} .7$ and an rms noise of $0.35 \mathrm{mJy}^{\text {beam }}{ }^{-1}$.

\section{RESOLVED IMAGES}

Figure 1 presents the CSO $350 \mu \mathrm{m}$ continuum image of HD 107146. The rms noise in the image is $6 \mathrm{mJy}^{\text {beam }}{ }^{-1}$, and the peak flux density $\left(181 \mathrm{mJy}^{\text {beam }}{ }^{-1}\right)$ is detected with a

\footnotetext{
7 http://www.iram.fr/IRAMFR/IS
}

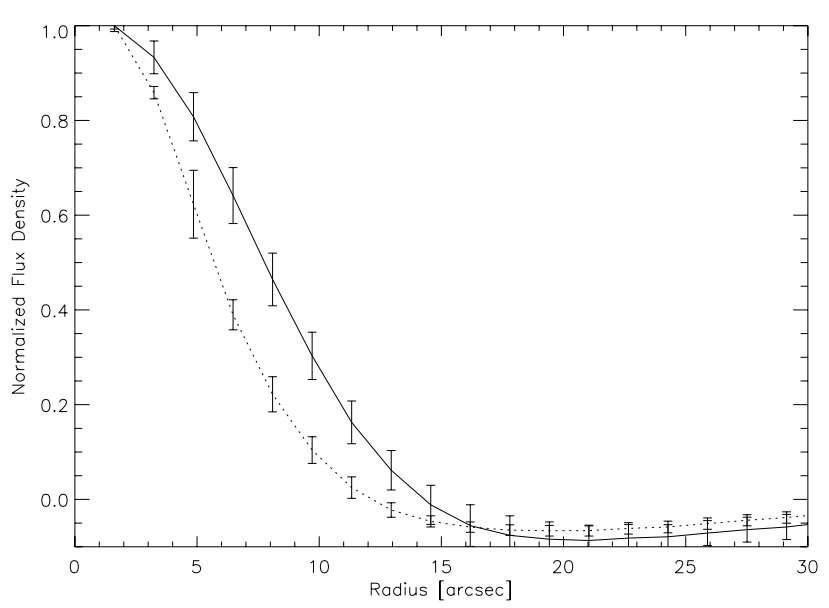

Figure 2. Radial profile of the CSO $350 \mu \mathrm{m}$ continuum emission for the HD 107146 debris disk (solid curve) and Callisto (dashed curve). Both radial profiles were measured on co-added images of the sources.

signal-to-noise ratio of 30 . The integrated $350 \mu \mathrm{m}$ flux density obtained by fitting an elliptical Gaussian to the image is $319 \pm$ $45 \mathrm{mJy}$, where the $1 \sigma$ uncertainty includes the statistical (6 mJy) and calibration ( $45 \mathrm{mJy}$ ) uncertainties added in quadrature. The centroid of the $350 \mu \mathrm{m}$ continuum emission is offset from the stellar position by $(\Delta \alpha, \Delta \delta)=\left(0,5,-1^{\prime \prime} .0\right)$; this offset is consistent within the pointing accuracy of the CSO telescope. No significant azimuthal structure in the $350 \mu \mathrm{m}$ emission is detected.

Figure 2 shows the azimuthally averaged radial profile of the $350 \mu \mathrm{m}$ emission for HD 107146 (solid curve) and Callisto (dashed curve). Callisto had an angular diameter of 1".49 at the time of the observations and is considered a point source for this analysis. The measured FWHM of the emission profile on Callisto is $9^{\prime \prime} .8 \pm 0.03$. By contrast, the FWHM size of the HD 107146 debris disk measured from an elliptical Gaussian fit to the $350 \mu \mathrm{m}$ image is $13^{\prime \prime} .5 \times 12^{\prime \prime} .6$. The deconvolved image size of HD 107146 using the observed Callisto image to represent the beam profile is $\left(8^{\prime \prime} .9 \pm 00^{\prime} .6\right) \times\left(8^{\prime \prime} .2 \pm 0.5\right)$. The $350 \mu \mathrm{m}$ continuum emission toward HD 107146 is clearly resolved.

Figure 3 presents the CARMA $1.3 \mathrm{~mm}$ continuum image of HD 107146 at $3^{\prime \prime}$ resolution. The $1.3 \mathrm{~mm}$ continuum emission extends over a $\sim 10^{\prime \prime}$ region. The integrated flux density obtained by summing the emission within a $12^{\prime \prime} \times 12^{\prime \prime}$ box centered on the stellar position is $10.4 \pm 1.4 \mathrm{mJy}$, with an additional $15 \%$ calibration uncertainty (Section 2).

The $1.3 \mathrm{~mm}$ emission is resolved into a partial ring that encircles the stellar position. The locations of the emission peaks are robust to various calibration schemes and data reduction approaches, but the magnitude of the peaks and the gap in the northwestern part of the ring are somewhat variable with such changes. The two brightest positions in the ring are located nearly equidistant from the stellar position on opposite sides of the star. The northeast peak is located 3".3 from the star at a position angle (P.A.; east of north) of P.A. $=48^{\circ}$, and the southwest peak is offset by 3".5 from the stellar position at P.A. $=190^{\circ}$. The projected distance of the dust peaks from the star is $\sim 97 \mathrm{AU}$.

The clumpy structure observed in the CARMA $1.3 \mathrm{~mm}$ continuum image is in contrast to the smooth, continuous ring observed in scattered light at optical wavelengths (Ardila et al. 2004). Further, the scattered-light image peaks at a larger orbital radius (130 AU) than the dust continuum image. The differences 


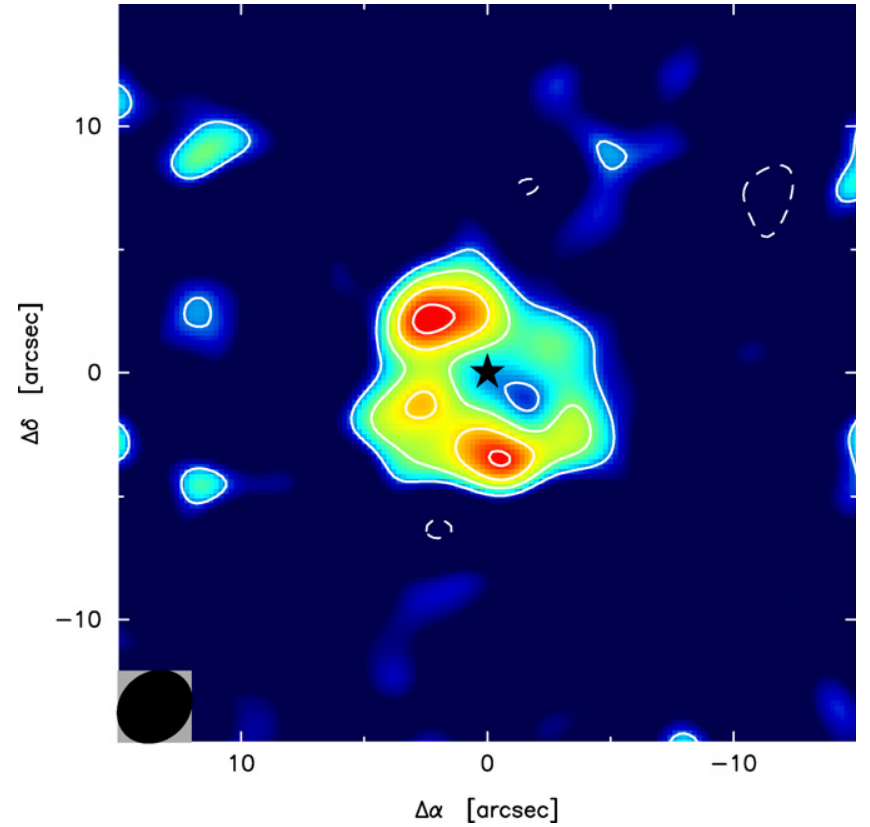

Figure 3. CARMA $1.3 \mathrm{~mm}$ continuum image of the HD 107146 debris disk. The FWHM of the synthesized beam $\left(3^{\prime \prime} .2 \times 2\right.$ ". 7$)$ is shown in the lower-left corner. Solid contours begin at $2 \sigma$ with increments of $1 \sigma$, where $\sigma=0.35 \mathrm{mJy} \mathrm{beam}^{-1}$ Dashed contours begin at $-2 \sigma$; no $<-3 \sigma$ pixel values are present within the region shown. The filled star at $(0,0)$ indicates the stellar position. The "dirty" beam has been deconvolved, but no primary beam corrections have been applied.

in the radial distribution is qualitatively consistent with the notion that small grains, which are efficient at scattering, are ejected by radiation pressure from the system, while the larger grains are found at smaller radii (Takeuchi \& Artymowicz 2001; Wyatt 2006).

The morphological differences of the $350 \mu \mathrm{m}$ and $1.3 \mathrm{~mm}$ images can be attributed to resolution in that if we produce the $1.3 \mathrm{~mm}$ CARMA image with a $8^{\prime \prime}$ FWHM taper to yield a $9^{\prime \prime}$ synthesized beam, the resulting image is centrally peaked on the stellar position and is similar in size to the $350 \mu \mathrm{m}$ image. In detail, other published, resolved, (sub-)millimeter images are inconsistent with the CSO and CARMA images. Williams et al. (2004) report that the $450 \mu \mathrm{m}$ emission at $8^{\prime \prime}$ resolution is offset from the stellar position by 4".4. Since the $350 \mu \mathrm{m}$ (see Figure 1) and the $850 \mu \mathrm{m}$ image (Williams et al. 2004) are centered on the stellar position to within $2^{\prime \prime}$, we assume that the offset in the $450 \mu \mathrm{m}$ image is a pointing error. A more significant discrepancy is seen between the CARMA $1.3 \mathrm{~mm}$ image and the OVRO $3 \mathrm{~mm}$ image (Carpenter et al. 2005). The $3 \mathrm{~mm}$ emission was resolved at $4 . .5 \times 4{ }^{\prime \prime} .0$ resolution with a FWHM size of $\left(6^{\prime \prime} .5 \pm 1^{\prime \prime} .4\right) \times\left(4^{\prime \prime} .2 \pm 1^{\prime \prime} .3\right)$. We have smoothed the $1.3 \mathrm{~mm}$ image to the resolution of the $3 \mathrm{~mm}$ image, and the ring structure is not apparent at the coarser resolution. However, the apparent ellipticity in the $3 \mathrm{~mm}$ image is nearly orthogonal to the clumps in the $1.3 \mathrm{~mm}$ image. Since the $1.3 \mathrm{~mm}$ image has higher signal to noise, finer resolution, and more baselines for better imaging, we view that image as a more reliable tracer of the debris structure.

\section{IMPLICATIONS}

Assuming gas drag is negligible, the orbital lifetime of dust grains in an optically thin disk is limited by Poynting-Robertson drag, collisional grinding of particles combined with radiation blowout of small grains, and stellar-wind drag. Dominik \& Decin (2003) have shown that for debris disks detectable with current instrumentation, collisional grinding of particles to the radiation blowout size dominates over Poynting-Robertson drag in removing mass from the debris system. Stellar-wind drag may also be important (Gustafson 1994), but the magnitude of this effect is poorly constrained at young ages (Jura 2004; Wood et al. 2005).

If a collisional cascade has produced the dust particles in the HD 107146 debris disk, the expected particle size distribution is $n(a) \propto a^{-3.5}$ (Dohnanyi 1969) for grain sizes larger than the radiation blowout size (radius $\sim 0.5 \mu \mathrm{m}$ for a solar-type star). In practice, the truncated distribution will produce a "wavy" pattern of particle sizes superimposed on the power law (e.g., Thébault \& Augereau 2007), but we neglect that complication. For silicate grains (Weingartner \& Draine 2001) larger than the blowout size, half of the flux density at a wavelength of $1.3 \mathrm{~mm}$ will be emitted by grains larger than $\sim 0.5 \mathrm{~mm}$ in radius for an $a^{-3.5}$ size distribution. The ratio of radiation to gravitational forces $(\beta)$ for these particles is $\beta \sim 0.001$, indicating radiation pressure has a negligible effect on their dynamics.

By the above arguments, the millimeter-wave emission traces predominantly millimeter-sized particles where the dynamics are controlled by particle collisions and gravity. This is consistent with the clumpy structure observed in the debris disk, as radiation forces will tend to produce a smooth spatial distribution of particles. The millimeter-wave emission should then trace the location of the planetesimals in the HD 107146 disk (Wyatt 2006). The radius of the observed debris emission implies the presence of planetesimals at an orbital radius of $\sim 97$ AU. Moreover, the dual emission peaks in the CARMA image presents intriguing possibilities for the presumed planetary system around HD 107146. The dust emission peaks are distributed nearly (but not precisely) symmetrically around the star, and may be caused by dust trapped in mean-motion resonance with an orbiting star or planet. In fact, the image of HD 107146 looks quite similar to the $\beta \sim 0.002,3: 2$ resonance image of Wyatt (2006). In detail, the two main dust peaks and the star are not collinear, which may imply a planet of moderate eccentricity $(e \sim 0.5)$ has trapped the dust in a 3:1 resonance (Kuchner \& Holman 2003). Given that the clumps are located at $97 \mathrm{AU}$, such resonances would imply a planet between $\sim 45$ and 75 AU.

Metchev \& Hillenbrand (2003) obtained adaptive optics images at $\lambda=2.2 \mu \mathrm{m}$ to search for companions to HD 107146. They place an upper mass limit of $10 M_{\mathrm{J}}$ to any companions at 74 AU. Apai et al. (2008) report that a similar limit of $10 \mathrm{M}_{\mathrm{J}}$ persists inward to $\sim 25 \mathrm{AU}$. Thus these observations rule out the presence of a stellar companion at such a large radius, and suggest that the structure in the debris disk is produced from the gravitational affects of a planetary system.

Could a planet have formed at $\sim 45-75$ AU that produced resonant structure in the debris disk? Kenyon \& Bromley (2004a) have simulated the collision growth of planets and subsequent production of debris and find that planets on the size of $\sim 2000 \mathrm{~km}\left(\sim 0.01 M_{\oplus}\right)$ are needed to excite the collisional cascade. The timescale to produce bodies of this size at 4575 AU from collisional growth of planetesimals is $\sim 50$ 225 Myr (Kenyon \& Bromley 2004a) for planetesimal with low eccentricity $(e<0.02)$ and a surface density profile appropriate for the minimum mass solar nebula (Weidenschilling 1977). For moderate eccentricities $(e \sim 0.02-0.04)$, the timescales are 2-4 times longer. Similarly, Kenyon \& Bromley (2004b) simulated the collisional growth of objects at 40-47 AU and found that $1000 \mathrm{~km}$ sized objects can form in 10-50 Myr, and double in mass every $100 \mathrm{Myr}$ to $1 \mathrm{Gyr}$. Thus the timescale to form 
$\sim 2000 \mathrm{~km}$ sized bodies, which are needed to initiate the collisional cascade, is roughly consistent with the age estimates of HD 107146.

However, the planet is likely much larger than $2000 \mathrm{~km}$ in radius if the dust is trapped in a resonance. Roques et al. (1994) conducted numerical simulations to show that planets more massive than $\sim 5 M_{\oplus}(\sim 11,000 \mathrm{~km}$ in radius assuming a volume density comparable to the Earth) are needed to trap particles in outer mean motion resonances. The timescale to grow such objects by collisional growth is more than $1 \mathrm{Gyr}$ at radii $>45 \mathrm{AU}$ (Kenyon \& Bromley 2004b), which is substantially older than HD 107146.

The presence of a massive planet at large radii then requires formation mechanisms beyond collisional aggregation of planetesimals. For disk-to-star mass ratios of $\geqslant 0.1$ in the $T$ Tauri phase, marginally consistent with observations (Andrews \& Williams 2007), planets may form by gravitational instability (e.g., Boss 2008, and references therein). Planets formed by this mechanism tend to be quite massive ( $1 M_{\mathrm{J}}$ or larger). Rafikov (2005) argues that the cooling timescales are inconsistent with formation via this method unless the planet is both massive, $\sim 5-10 M_{\mathrm{J}}$, and distant, $\sim 100 \mathrm{AU}$, which is consistent with the distance and upper mass limit described above. Another possibility is that the planet formed in the inner regions and migrated outward, which may trap planetesimals and dust in resonant orbits (Wyatt 2003). Such a mechanism has been used to explain the structure and dynamics of the Kuiper Belt (Malhotra 1993, 1995). Detailed modeling is needed to gain more insights on the planetary system around HD 107146 and if migration can explain the clumpy structure observed in the surrounding debris disk.

In summary, the young solar analog HD 107146 is surrounded by a clumpy debris disk similar to those seen around Vega and $\epsilon$ Eridani. The structure is suggestive of dynamical influence from a planet. The likely size and location of the presumed planet are inconsistent with formation by purely collisional aggregation of planetesimals and appeals to migration or formation via gravitational instability must be made.

We thank the referee for comments which improved this work. Support for CARMA construction was derived from the Gordon and Betty Moore Foundation, the Kenneth T. and Eileen L. Norris Foundation, the Associates of the California Institute of Technology, the states of California, Illinois, and Maryland, and the National Science Foundation. Ongoing CARMA development and operations are supported by the National Science Foundation under a cooperative agreement, and by the CARMA partner universities.

\section{REFERENCES}

Altenhoff, W. J., Thum, C., \& Wendker, H. J. 1994, A\&A, 281, 161

Andrews, S. M., \& Williams, J. P. 2007, ApJ, 659, 705

Apai, D., et al. 2008, ApJ, 672, 1196

Ardila, D. R., et al. 2004, ApJ, 617, L147

Boss, A. P. 2008, ApJ, 677, 607

Carpenter, J. M., Wolf, S., Schreyer, K., Launhardt, R., \& Henning, T. 2005, AJ, 129, 1049

Clark, B.G. 1980, A\&A, 89, 377

Dohnanyi, J. W. 1969, J. Geophys. Res., 74, 2531

Dominik, C., \& Decin, G. 2003, ApJ, 598, 626

Dowell, C. D., et al. 2003, Proc. SPIE, 4855, 73

Greaves, J. S., et al. 1998, ApJ, 506, L133

Greaves, J. S., et al. 2005, ApJ, 619, L187

Gustafson, B. A. S. 1994, Annu. Rev. Earth Planet. Sci., 22, 553

Högbom, J. A. 1974, A\&AS, 15, 417

Holland, W. S., et al. 1998, Nature, 392, 788

Jaschek, M. 1978, Bull. Inf. Centre Donnees Stellaires, 15, 121

Jura, M. 2004, ApJ, 603, 729

Kalas, P., Liu, M. C., \& Matthews, B. C. 2004, Science, 303, 1990

Kalas, P., Graham, J. R., \& Clampin, M. 2005, Nature, 435, 7045

Kenyon, S. J., \& Bromley, B. C. 2004a, AJ, 127, 513

Kenyon, S. J., \& Bromley, B. C. 2004b, AJ, 128, 1916

Koerner, D. W., Sargent, A. I., \& Ostroff, N. A. 2001, ApJ, 560, L181

Kovacs, A. 2008, Proc. SPIE, 7020 (arXiv:0805.3928)

Kuchner, M. J., \& Holman, M. J. 2003, ApJ, 588, 1110

Liou, J.-C., \& Zook, H. A. 1999, AJ, 118, 580

Malhotra, R. 1993, Nature, 365, 819

Malhotra, R. 1995, AJ, 110, 420

Maness, H., Fitzgerald, M. P., Paladini, R., Kalas, P., Duchene, G., \& Graham, J. R. 2008, ApJ, 686, 25

Metchev, S. A., \& Hillenbrand, L. A. 2003, in ASP Conf. Ser. 324, Debris Disks and the Formation of Planets, ed. L Caroff \& D. Backman (San Francisco, CA: ASP)

Meyer, M. R., Backman, D. E., Weinberger, A. J., \& Wyatt, M. C. 2007, in Protostars and Planets V, ed. B. Reipurth, D. Jewitt, \& K. Keil (Tucson, AZ: Univ. Arizona Press)

Perryman, M. A. C., et al. 1997, A\&A, 323, L49

Rafikov, R. R. 2005, ApJ, 621, L69

Roques, F., Scholl, H., Sicardy, B., \& Bradford, A. S. 1994, Icarus, 108, 37

Sault, R. J., Teuben, P. J., \& Wright, M. C. H. 1995, ASP Conf. Ser. 77, Astronomical Data Analysis Software and Systems IV (San Francisco, CA: ASP), 433

Schneider, G., et al. 2006, ApJ, 650, 414

Steer, D. G., Dewdney, P. E., \& Ito, M. R. 1984, A\&A, 137, 159

Takeuchi, T., \& Artymowicz, P. 2001, ApJ, 557, 990

Thébault, P., \& Augereau, J.-C. 2007, A\&A, 472, 169

Weidenschilling, S. J., Ap\&SS, 51, 153

Weingartner, J. C., \& Draine, B. T. 2001, ApJ, 548, 296

Williams, J. P., Najita, J., Liu, M. C., Bottinelli, S., Carpenter, J. M., Hillenbrand, L. A., Meyer, M. R., \& Soderblom, D. R. 2004, ApJ, 604, 414

Wilner, D. J., Holman, M. J., Kuchner, M. J., \& Ho, P. T. P. 2002, ApJ, 597, L115

Wood, B. E., Müller, H.-R., Zank, G. P., Linsky, J. L., \& Redfield, S. 2005, ApJ, $628, \mathrm{~L} 143$

Wyatt, M. C. 2003, ApJ, 598, 1321

Wyatt, M. C. 2006, ApJ, 639, 1153 